\title{
REMARKS ON THE NUMBER OF PRIME DIVISORS OF INTEGERS
}

\author{
MEHDI HASSANI
}

Abstract. In this paper, we obtain explicit bounds for sums $\sum_{k \leqslant n} \omega(k)$ and $\sum_{k \leqslant n} \Omega(k)-\omega(k)$, where $\omega(k)$ denotes the number of distinct prime divisors of $k$, and $\Omega(k)$ denotes the total number of its prime divisors. Moreover, we give some better explicit bounds for the sum $\sum_{k \leqslant n} \omega(k)$ under assumption of the Riemann hypothesis.

Mathematics subject classification (2010): 11A41, 11A25, 26D20.

Keywords and phrases: Primes, arithmetic functions, inequalities.

\section{REFERENCES}

[1] R. L. Duncan, A class of additive arithmetical functions, Amer. Math. Monthly 69 (1962), 34-36.

[2] S. R. Finch, Mathematical constants, Encyclopedia of Mathematics and its Applications, 94, Cambridge University Press, Cambridge, 2003.

[3] G. HARDY AND S. RAMANUJAN, The normal number of prime factors of a number $n$, Quart. J. Math. 48 (1917), 76-92.

[4] M. HASSANI, Factorization of factorials and a result of Hardy and Ramanujan, Mathematical Inequalities \& Applications, 15, 2 (2012), 403-407.

[5] J. B. Rosser \& L. Schoenfeld, Approximate Formulas for Some Functions of Prime Numbers, Illinois J. Math. 6 (1962), 64-94.

[6] L. Schoenfeld, Sharper Bounds for the Chebyshev Functions $\theta(x)$ and $\psi(x)$. II, Math. of Computation 30, 134 (April 1976), 337-360. 